\title{
Whole slide imaging in pathology: what is holding us back?
}

This article was published in the following Dove Press journal:

Pathology and Laboratory Medicine International

II June 2015

Number of times this article has been viewed

\section{Michael D Feldman \\ Department of Pathology, Perelman School of Medicine at the University of Pennsylvania School of Medicine, Philadelphia, PA, USA}

Despite all the advances and improvements discussed in the article by Pantanowitz et al in this journal, the clinical adoption of whole slide imaging (WSI) for primary diagnostics has only shown limited penetration outside the USA, while practitioners in the USA await US Food and Drug Administration (FDA) approval to use WSI for primary diagnostics. Areas with shortages of pathologists or where subspecialty expertise are not available have seen adoption ${ }^{1-3}$ for use in frozen section diagnostic support in neuropathology as well as other subspecialized disciplines. More recently, international consultation between countries with smaller numbers of pathologist or subspecialist access have been using telepathology WSI for consultations in the USA, Canada, or Europe. In Sweden, more widespread adoption of WSI for primary diagnostics has occurred, but even there, pathologists still use a mix of WSI and microscopes for primary diagnostics. ${ }^{4}$ Why, despite all the advances described in the accompanying article, has WSI not taken pathology by storm like digital radiology and picture archiving and communication system (PACS) took radiology by storm years ago. Where have we fallen short and why has there been slow adoption of WSI beyond the research and education venues, where it has been much more broadly adopted. Perhaps because the use cases in research and education have such a strong return on investment (ROI), they have been much better targets for early adoption of WSI.

It has been more than 10 years since the first rapid high speed scanners for WSI came onto the market. While these high speed scanners have improved and new approaches to WSI have evolved, today's scanners provide high spatial and color fidelity in the $\mathrm{X}-\mathrm{Y}$ plane but they still cannot recapitulate all the functions of a standard clinical microscope. Today's scanners cannot perform a Z-axis across an entire slide rapidly. While some scanners are capable of a Z-stack scan, these scanners acquire the Z-stack by making multiple individual scans at different focal planes, a very time consuming process that makes them impractical for widespread use of a Z-stack in today's high volume clinical labs. How important are multiple focal planes? Without a side by side study it is impossible to say, but consider the types of uses and frequency that we use the Z-axis on our microscopes. Without multiple focal planes, accurately assessing mitotic figures are only accurate to $80 \%-85 \%$ as described in the accompanying article and this point was further demonstrated in a software competition where image scientists were asked to create algorithms that could detect mitoses and the best the advanced image processing software were able to achieve was a similar $80 \%-85 \%$ accuracy compared to manually annotated slides using an analog microscope. ${ }^{5-7}$ Multiple focal
Correspondence: Michael D Feldman Department of Pathology, University of Pennsylvania Health System, Founders building 6th floor, Room 6067, 3400 Spruce Street, Philadelphia, PA 19104, USA

Email feldmanm@mail.med.upenn.edu 
planes are widely used beyond mitoses counting and are frequently used in evaluation of micro-organisms, evaluation of nuclear chromatin texture, and nuclear contour irregularities in both surgical pathology and cytopathology. In cytopathology, no one would imagine practicing cytopathology without a Z-axis to analyze the three dimensional contours of cells and tissue fragments. In short, we use the Z-axis in many clinical scenarios, yet none of today's scanners are able to capture this multifocal plane data rapidly and reliably. As described in the accompanying article, new advances in WSI have been recently described which hold promise for rapidly capturing a WSI with a full Z-axis, ${ }^{8}$ however, until this technology becomes widely available, practitioners of WSI will be significantly limited without an important functional tool that is routinely available on their analog microscope.

While great progress has been made in areas of image acquisition, with current scan times routinely approaching 60 seconds for a WSI image, the clinical sign-out process using WSI still takes longer than using a microscope. Randell et $\mathrm{al}^{9-11}$ demonstrated that digital pathology in its current form and with current viewing software and monitors is $60 \%$ slower on average than a microscope. They examined multiple tasks a pathologist routinely performs, such as making a diagnosis, deciding if a lymph node has metastatic tumor in it, finding rare events on a slide (microorganism or isolated tumor cells), navigate to a specific area of a slide, and attempt a diagnosis on a challenging case. All five of these commonly performed tasks we do every day with our analog microscope was slower using current WSI technology. Two other microscope tasks (making a measurement on a slide and scoring a tissue microarray) measured were roughly equivalent. They went on to show the complexity of our diagnostic process. They demonstrated that we frequently multitask while looking at slides in our diagnostic sessions. We navigate paper requisitions, create reports, fill out synoptic summaries, annotate slides with pens, and search for additional clinical or radiologic data to help us understand the tissue process we are evaluating. They went on to show that with a proper user interface design for slide viewing, digital pathology could be brought on par with the speed of an analog microscope. But is on par fast enough or do we want a system that is faster and more efficient than today's analog microscope? At a time of diminishing revenue and greater demands, pathologists will be asked to do more without commensurate increases in staffing. Systems and tools that allow enhanced capability will be needed but are not currently available in today's market.
Many of the software tools described in the accompanying article are widely used for multiple functions, such as scoring in breast cancer (estrogen receptor, progesterone receptor, and Her2) and quantitating proliferating cells (Ki67). These quantitative immunohistochemical scoring algorithms have proven to be very useful and have been widely implemented across community, academic, and commercial pathology practices. However, in the grand scale of all the work we do in pathology, quantitative scoring of immunohistochemistry represents a very small percentage of our total work effort. What is lacking are new image analytic tools and paradigms to adopt and deploy these tools to enable and enhance how pathologists perform their work on a routine daily basis. Several groups have been experimenting with content based image retrieval systems ${ }^{12,13}$ and several large companies including Google, Microsoft, and IBM have active programs in image query, but none of these have found their way into routine pathology workflow. Several investigators have demonstrated promising capability of advanced image analytics, including the ability to predict biologic behavior of tumors in areas of breast pathology, oropharyngeal squamous cell carcinoma, and prostate carcinoma. ${ }^{14,15}$ Several groups have begun to explore and implement algorithms using deep learning methods with very promising results in the areas of tumor segmentation, mitoses detection, and tumor aggressivity. ${ }^{7,16}$ This scholarly work points to a very exciting future for new paradigms and practice capabilities. However, these algorithms are still in our research laboratories in development and need further validation against larger cohorts linked to long-term clinical outcomes before they will be accepted for routine clinical use.

WSI has found widespread adoption in the pharmaceutical industry as it has allowed companies to speed up the toxicologic analysis of the preclinical phase of drug discovery. It has accomplished this by marrying WSI slides with associated digital metadata captured from the slides with biorepository and Clinical Trials laboratory information management systems. These systems then allow distribution of material to networks of geographically dispersed pathologist or replace the pathologist through automation of scoring previously done manually and now facilitated by advanced image processing algorithms. These image analytic tools allow veterinary pathologists to more quickly review and score cases for many features including fibrosis, steatosis, necrosis, apoptosis, or enumerate quantitative immunohistochemistry. ${ }^{17,18}$ Speed is valuable in the pharmaceutical industry and any innovation that compresses time in the drug pipeline and helps to identify drugs which can move past the preclinical toxicity 
stage or cannot move past the preclinical stage has a very positive ROI.

Similar to pharmacology, medical schools, veterinary schools, and dental schools have seen widespread adoption of WSI in teaching histology and pathology to their students because of the tight linkage to a positive ROI and ease of use of WSI for teaching compared to analog microscopes. Schools no longer need to manage and maintain hundreds of microscopes and glass slide collections (eliminating slide breakage or issues of uneven slide quality), and students are no longer tethered to the microscopy lab at their school of medicine for study. Today students study in groups across multiple spaces at convenient times using WSI systems housed at their school or set up in the cloud. The initial cost of these systems is less than the cost of outfitting a school with microscopes for all their students, and student adoption and engagement using WSI has been widely accepted and appreciated by students. ${ }^{19-21}$

The business case for WSI in clinical practice has been a much more challenging process. Since existing WSI systems do not make the pathologist any faster, the ROI on these systems has been difficult to justify. Adoption has been focused on health care systems or countries with "geographic use" cases where the health system does not have access to an individual pathologist or a subspecialist pathologist in a geographically diverse region. ${ }^{1,222-24}$ The business case for WSI adoption for primary diagnostics is still evolving and despite publications which suggest a large positive ROI, no health system in the USA or Europe has seen this ROI. ${ }^{25}$ The innovations that are occurring in WSI scanners, image analytics, and user interface design need to be accompanied by health system business innovation that creatively thinks about how diagnostic medicine is practiced today and how it may be practiced in the future.

The future of digital pathology is bright but it must be thought about as a total system (from specimen through grossing to slide creation, scanning, review, and reporting) with each component playing a vital role in the process. We are gradually reaching a critical mass and critical functions in many of these areas of the process. With each step in the process providing opportunities for innovation, our pathology process will undoubtedly change and migrate to a full digital workflow but several of the hurdles defined in this editorial need to be tackled before that becomes a reality.

\section{Disclosure}

MDF acts as a consultant and is a member of the scientific advisory board for Inspirata Inc., Tampa, FL and is also on the scientific advisory board for Xifin Inc., San Diego, CA. The author reports no other conflicts of interest in this work.

\section{References}

1. Canadian Association of Pathologists Telepathology Guidelines Committee, Bernard C, Chandrakanth SA, et al. Guidelines from the Canadian Association of Pathologists for establishing a telepathology service for anatomic pathology using whole-slide imaging. J Pathol Inform. 2014;5:15.

2. Evans AJ, Kiehl TR, Croul S. Frequently asked questions concerning the use of whole-slide imaging telepathology for neuropathology frozen sections. Semin Diagn Pathol. 2010;27(3):160-166.

3. Pantanowitz L, Valenstein PN, Evans AJ, et al. Review of the current state of whole slide imaging in pathology. J Pathol Inform. 2011;2:36.

4. Thorstenson S, Molin J, Lundstrom C. Implementation of large-scale routine diagnostics using whole slide imaging in Sweden: Digital pathology experiences 2006-2013. J Pathol Inform. 2014;5:14.

5. Al-Janabi S, van Slooten HJ, Visser M, van der Ploeg T, van Diest PJ, Jiwa M. Evaluation of mitotic activity index in breast cancer using whole slide digital images. PloS One. 2013;8(12):e82576.

6. Roux L, Racoceanu D, Lomenie N, et al. Mitosis detection in breast cancer histological images An ICPR 2012 contest. J Pathol Inform. 2013;4:8.

7. Veta M, van Diest PJ, Willems SM, et al. Assessment of algorithms for mitosis detection in breast cancer histopathology images. Med Image Anal. 2015;20(1):237-248.

8. Zheng G, Horstmeyer R, Yang C. Wide-field, high-resolution Fourier ptychographic microscopy. Nat Photonics. 2013;7(9):739-745.

9. Randell R, Ambepitiya T, Mello-Thoms C, et al. Effect of display resolution on time to diagnosis with virtual pathology slides in a systematic search task. J Digit Imaging. 2015;28(1):68-76.

10. Randell R, Ruddle RA, Mello-Thoms C, Thomas RG, Quirke P, Treanor D. Virtual reality microscope versus conventional microscope regarding time to diagnosis: an experimental study. Histopathology. 2013;62(2):351-358.

11. Randell R, Ruddle RA, Quirke P, Thomas RG, Treanor D. Working at the microscope: analysis of the activities involved in diagnostic pathology. Histopathology. 2012;60(3):504-510.

12. Akakin HC, Gurcan MN. Content-based microscopic image retrieval system for multi-image queries. IEEE Trans Inf Technol Biomed. 2012; 16(4):758-769.

13. Zheng L, Wetzel AW, Gilbertson J, Becich MJ. Design and analysis of a content-based pathology image retrieval system. IEEE Trans Inf Technol Biomed. 2003;7(4):249-255.

14. Beck AH, Sangoi AR, Leung S, et al. Systematic analysis of breast cancer morphology uncovers stromal features associated with survival. Sci Transl Med. 2011;3(108):108ra113.

15. Basavanhally A, Feldman M, Shih N, et al. Multi-field-of-view strategy for image-based outcome prediction of multi-parametric estrogen receptor-positive breast cancer histopathology: Comparison to Oncotype DX. J Pathol Inform. 2011;2:S1.

16. Cruz-Roa AA, Arevalo Ovalle JE, Madabhushi A, Gonzalez Osorio FA. A deep learning architecture for image representation, visual interpretability and automated basal-cell carcinoma cancer detection. Med Image Comput Comput Assist Interv. 2013;16(Pt 2):403-410.

17. Shinde V, Burke KE, Chakravarty A, et al. Applications of pathologyassisted image analysis of immunohistochemistry-based biomarkers in oncology. Vet Pathol. 2014;51(1):292-303.

18. Webster JD, Dunstan RW. Whole-slide imaging and automated image analysis: considerations and opportunities in the practice of pathology. Vet Pathol. 2014;51(1):211-223.

19. Dee FR, Meyerholz DK. Teaching medical pathology in the twenty-first century: virtual microscopy applications. J Vet Med Educ. 2007;34(4): 431-436. 
20. Heidger PM Jr, Dee F, Consoer D, Leaven T, Duncan J, Kreiter C. Integrated approach to teaching and testing in histology with real and virtual imaging. Anat Rec. 2002;269(2):107-112.

21. Kumar RK, Velan GM, Korell SO, Kandara M, Dee FR, Wakefield D. Virtual microscopy for learning and assessment in pathology. J Pathol. 2004;204(5):613-618.

22. Evans AJ, Chetty R, Clarke BA, et al. Primary frozen section diagnosis by robotic microscopy and virtual slide telepathology: the University Health Network experience. Semin Diagn Pathol. 2009;26(4):165-176.
23. Tetu B, Fortin JP, Gagnon MP, Louahlia S. The challenges of implementing a "patient-oriented" telepathology network; the Eastern Quebec telepathology project experience. Anal Cell Pathol (Amst). 2012;35(1):11-18.

24. Tetu B, Perron E, Louahlia S, Pare G, Trudel MC, Meyer J. The Eastern Quebec Telepathology Network: a three-year experience of clinical diagnostic services. Diagn Pathol. 2014;9 Suppl 1:S1.

25. Ho J, Ahlers SM, Stratman C, et al. Can digital pathology result in cost savings? A financial projection for digital pathology implementation at a large integrated health care organization. J Pathol Inform. 2014;5:33.

\section{Publish your work in this journal}

Pathology and Laboratory Medicine International is a peer-reviewed, open access journal focusing on innovative basic research and translational research related to pathology or human disease. The journal includes original research, updates, case reports, reviews and commentaries on current controversies. The Academic Sponsor of this journal is the Chinese American Pathology Association (CAPA). The manuscript management system is completely online and includes a very quick and fair peer-review system. Visit http://www.dovepress.com/testimonials.php to read real quotes from published authors.

Submit your manuscript here: http://www.dovepress.com/pathology-and-laboratory-medicine-international-journal 